\title{
NUMERICAL DETERMINATION OF VOLTAGE POTENTIAL INSIDE NONHOMOGENEOUS MEDIA USING VARIATIONAL METHODS
}

\author{
Igor BRILLA*, František JANÍČEK ${ }^{* *}$ \\ *Department of Mathematics, Faculty of Electrical Engineering and Information Technology, Slovak University of Technology, \\ Ilkovičova 3, Bratislava, Slovak Republic, tel. +421 260291 332, E-mail: igor.brilla@stuba.sk \\ ${ }^{* *}$ Institute of Power and Applied Electrical Engineering, Faculty of Electrical Engineering and Information Technology, Slovak \\ University of Technology, Ilkovičova 3, Bratislava, Slovak Republic, tel. +421 260291 298, E-mail: frantisek.janicek@stuba.sk
}

\begin{abstract}
The problem of numerical determination of voltage potential inside one and two dimensional nonhomogeneous media using values of the voltage potential on the boundary of the media has been solved. The convenient form of variational formulation of the problem has been derived. The numerical solution of the problem has been obtained by the method of local variations.
\end{abstract}

Keywords: voltage potential, numerical determination, local variations

\section{INTRODUCTION}

The paper is dealt with the numerical determination of voltage potential inside one and two dimensional nonhomogeneous media using values of the voltage potential on the boundary of the media. For numerical determination of the problem is used the model described by the potential equation for the nonhomogeneous media with appropriate boundary conditions. The convenient form of variational formulation of the problem is derived. For numerical analysis of the problem are applied discrete methods. They are very convenient because in the case of practical problems input data are measured in discrete points. The numerical solution of the problem is obtained by the method of local variations [1]. The convergence of the method of local variations for the problem is proved.

The numerical experiments are done from a mathematical point of view. The numerical solutions are computed for different values of electrical conductivities of the media and for different values of the voltage potential on the boundary of the media. The case of discontinuous electrical conductivities is also considered.

\section{ONE DIMENSIONAL NONHOMOGENEOUS PROBLEM}

At first is considered one dimensional nonhomogeneous problem.

\subsection{Formulation of the problem}

The following one dimensional potential equation is considered

$-\left(c u^{\prime}\right)^{\prime}=0$ in $\Omega$,

where $c$ is an electrical conductivity of the medium, $u$ is a voltage potential, $\Omega$ is assumed as an one dimensional domain of following form $\Omega=\{x \in R: 0<x<a\}$. The following Dirichlet boundary conditions are considered for the voltage potential $u(0)=f(0), \quad u(a)=f(a)$,

where $f$ represents values of the voltage potential on the boundary of the domain $\Omega$.

It is possible to derive similarly as in [2] the convenient form of variational formulation of the problem (1), (2) as the following functional

$J(w)=\int_{a}^{b}\left[c\left(w^{\prime}\right)^{2}+2 c w^{\prime} v^{\prime}\right] d x$

where

$u=w+v$ in $\bar{\Omega}$

and

$v(0)=u(0), \quad v(a)=u(a)$.

It means that $v$ is arbitrary function having the same values on the boundary like $u$ and $w$ is fulfilling homogeneous boundary conditions

$w(0)=0, \quad w(a)=0$.

It is possible to show [2] that the solution of the problem (1), (2) may be characterized as stationary point $w$ of the functional (3) with respect to (4) - (6).

\subsection{Method of local variations}

Discrete methods are applied for numerical analysis of the problem. On the domain $\Omega$ is considered uniform grid $(m+1) h, h>0$. The functional $J$ is assumed to have the following discrete form

$J \approx I=h \sum_{i=0}^{m} I_{i}$

where 
$I_{i}=c_{i}^{*}\left[\left(w^{\prime}\right)_{i}^{*}\right]^{2}+2 c_{i}^{*}\left(w^{\prime}\right)_{i}^{*}\left(v^{\prime}\right)_{i}^{*}$

and

$$
\begin{aligned}
& c_{i}^{*}=\frac{c_{i}+c_{i+1}}{2}, \quad\left(w^{\prime}\right)_{i}^{*}=\frac{w_{i+1}-w_{i}}{h}, \\
& \left(v^{\prime}\right)_{i}^{*}=\frac{v_{i+1}-v_{i}}{h}
\end{aligned}
$$

with the following discrete form of boundary conditions (6) and (5)

$$
\begin{aligned}
& w_{0}=w_{m+1}=0, \quad v_{0}=f(0), \\
& v_{m+1}=f(a) .
\end{aligned}
$$

Instead of finding the minimum of the functional $J$ now the problem arises to find values $w_{i}, i=0,1, \ldots, m+1$ which fulfil the boundary conditions (9) and such that (7) is minimal. The minimum of (7) is found using the method of local variations [1]. The algorithm of this method is given by a sequence of iterations. It is necessary to start the algorithm with determination of some initial approximation of the values $w_{i}, i=0,1, \ldots, m+1$ such that the boundary conditions (9) are fulfilled. The iterative solution is obtained in such a way, that the iteration from the previous step is considered as the initial approximation and then it is necessary to go through all interior grid points in arbitrary order. At each grid point $(i h) i=1,2, \ldots, m$ is computed the value

$$
\Phi_{i}\left(w_{i}\right)=h \sum_{j=0}^{m} \chi_{j} I_{j}
$$

where

$$
\chi_{j}=\left\{\begin{array}{ll}
1, & I_{j}=I_{j}\left(w_{i}\right) \\
0, & I_{j} \neq I_{j}\left(w_{i}\right)
\end{array},\right.
$$

which is the collection of such $I_{j}$ from (7) which depends on the value $w_{i}$. According to (8) it is possible to rewrite (10) in the following form

$$
\Phi_{i}\left(w_{i}\right)=h \sum_{j=i-1}^{i} I_{j} .
$$

Then the value of $w_{i}$ is changed by little to the values $w_{i} \pm p$, where $p>0$ is the given step and two corresponding values $\Phi_{i}^{+}\left(w_{i}+p\right), \Phi_{i}^{-}\left(w_{i}-p\right)$ are computed. The new value of the solution at grid point $(i h)$ is chosen from the values $w_{i}, w_{i}+p, w_{i}-p$ and it is this one which corresponds to the minimum value of $\Phi_{i}\left(w_{i}\right), \Phi_{i}^{+}\left(w_{i}+p\right), \Phi_{i}^{-}\left(w_{i}-p\right)$. The iteration is finished after we have gone through all interior grid points. Then the value of $I$ is calculated for such solution and the process is repeated until the value of $I$ is decreasing. Then it is possible to continue by dividing the step.

We can prove similarly to [3] the following important theorem:

THEOREM. Let the electrical conductivity is piecewise smooth function. Then the method of local variations is convergent for the problem (1), (2).

The program realization of the method of local variations for the problem is elaborated in the programming language Fortran 77.

\subsection{Numerical solution of the problem}

Numerical experiments are done from a mathematical point of view. This means that at first is constructed the problem with the exact solution, afterwards is computed the numerical solution of this problem using the method of local variations and in the end the computed numerical solution is compared it with the exact one.

The numerical solutions were computed for different values of electrical conductivities of the media and for different values of the voltage potential on the boundary of the media. The following domain $\Omega=\langle 0,2\rangle$ is considered.

For the following voltage potential

$u=\frac{1}{e^{x}}$

the electrical conductivity

$c=e^{x}$

using (13) and the boundary conditions constructed from (12), using the program realization of the method of local variations in the Table 1 it is able to see the percentage of errors in the computed solutions in the second column with respect to the exact solutions of the meshes given in

\begin{tabular}{|c|c|c|}
\hline Mesh & Error $(\%)$ & $\begin{array}{c}\text { Number of } \\
\text { iterations }\end{array}$ \\
\hline 8 & $1.010^{-7}$ & 83 \\
\hline 12 & $1.410^{-7}$ & 82 \\
\hline 16 & $1.210^{-7}$ & 84 \\
\hline
\end{tabular}
the first column. In the third column are reported the numbers of iterations after which the numerical solution is obtained on the given mesh. From the results it is possible to see that very small errors are obtained for a course mesh.

Table 1 Numerical results for the problem (12), (13)

For another voltage potential

$u=\ln (x+1)$

the electrical conductivity 
$c=(x+1)$

as it is obvious from the Table 2 the accuracy of computation is not so good. This fact is caused by the discretization error, which is in this case greater than in previous case. From Table 2 it is seen that when the number of grid points increases, errors decrease.

Table 2 Numerical results for the problem (14), (15)

\begin{tabular}{|c|c|c|}
\hline Mesh & Error $(\%)$ & $\begin{array}{c}\text { Number of } \\
\text { iterations }\end{array}$ \\
\hline 8 & $2.110^{-1}$ & 133 \\
\hline 12 & $1.010^{-1}$ & 223 \\
\hline 16 & $6.310^{-2}$ & 295 \\
\hline
\end{tabular}

One way how to obtain better results in this case is make computations for more grid points. Obtained results can be seen in the Table 3. From the Table 3 can be seen that the errors decrease, however the number of iterations rapidly increase. Second way how to obtain better results is to use better discretization scheme.

Table 3 Numerical results for the problem (14), (15)

$\begin{array}{ccc}\text { Mesh } & \text { Error }(\%) & \begin{array}{c}\text { Number of } \\ \text { iterations }\end{array} \\ 32 & 1.710^{-2} & 717 \\ 64 & 4.610^{-3} & 1703 \\ 128 & 1.110^{-3} & 3765\end{array}$

We also deal with the case when the electrical conductivity is discontinuous

$$
\begin{aligned}
& c=e^{x}, x \leq \frac{2}{3}, \\
& c=3, x>\frac{2}{3} .
\end{aligned}
$$

Then for the voltage potential

$$
\begin{aligned}
& u=\frac{1}{e^{x}}, x \leq \frac{2}{3}, \\
& u=\frac{-x}{3}+\frac{1}{e^{\frac{2}{3}}}+\frac{2}{9}, x>\frac{2}{3}
\end{aligned}
$$

as it is obvious from the Table 4 the accuracy of computation is also now not so good. Also now this fact is caused by the discretization error, which is in this case greater than in the first case. From Table 4 it is seen that when the number of grid points increases, errors decrease.

Also now one way how to obtain better results in this case is make computations for more grid points. Obtained results can be seen in the Table 5. From the Table 5 also
Table 4 Numerical results for the problem (16), (17)

$\begin{array}{ccc}\text { Mesh } & \text { Error (\%) } & \begin{array}{c}\text { Number of } \\ \text { iterations }\end{array} \\ 8 & 1.6 & 242 \\ 12 & 1.4 & 407 \\ 16 & 2.7 \quad 10^{-1} & 525\end{array}$

now can be seen that the errors slightly decrease, however the number of iterations rapidly increase. Also now

\begin{tabular}{|c|c|c|}
\hline Mesh & Error $(\%)$ & $\begin{array}{c}\text { Number of } \\
\text { iterations }\end{array}$ \\
\hline 32 & $3.210^{-1}$ & 2037 \\
\hline 64 & $4.310^{-2}$ & 5208 \\
\hline 128 & $7.510^{-2}$ & 22848 \\
\hline
\end{tabular}
second way how to obtain better results is to use better discretization scheme.

Table 5 Numerical results for the problem (16), (17)

\section{TWO DIMENSIONAL NONHOMOGENEOUS PROBLEM}

Now is considered two dimensional nonhomogeneous problem.

\subsection{Formulation of the problem}

The following two dimensional potential equation is considered

$-\left(c u_{, i}\right)_{, i}=0$ in $\Omega$,

where also now $c$ is an electrical conductivity of the medium, $u$ is a voltage potential. The summation and differentiation rule with respect to indices is applied. $\Omega$ is assumed as a two dimensional domain of following form $\Omega=\left\{(x, y) \in R^{2}: 0<x<a, 0<y<b\right\}$. The following Dirichlet boundary condition is considered for the voltage potential

$u(s)=f(s), \quad s \in \partial \Omega$,

where $f$ represents values of the voltage potential on the boundary of the domain $\Omega$.

The equation (18) for the two dimensional case can be written in the following form

$\left(c u_{, x}\right)_{, x}+\left(c u_{, y}\right)_{, y}=0 \quad$ in $\Omega$.

It is possible to derive similarly as in [2] the convenient form of variational formulation of the problem (20), (19) as the following functional 
$J(w)=\iint_{\Omega}\left[c w_{, x}^{2}+c w_{, y}^{2}+\right.$

$\left.+2 c w_{, x} v_{, x}+2 c w_{, y} v_{, y}\right\rfloor d x d y$

where

$u=w+v \quad$ in $\bar{\Omega}$

and

$v(s)=u(s), \quad s \in \partial \Omega$

It means that $v$ is arbitrary function having the same values on the boundary like $u$ and $w$ is fulfilling homogeneous boundary condition

$w(s)=0, \quad s \in \partial \Omega$.

It is possible to show [2] that the solution of the problem (20), (19) may be characterized as stationary point $w$ of the functional (21) with respect to $(22)-(24)$.

\subsection{Method of local variations}

Discrete methods are applied also now for numerical analysis of the problem. They are very convenient because in the case of practical problems input data are measured in discrete points. On the domain $\Omega$ is considered uniform grid $(m+1) h \times(n+1) h, h>0$. The functional $J$ assume following discrete form

$J \approx I=h^{2} \sum_{i=0}^{m} \sum_{k=0}^{n} I_{i ; k}$

where

$$
\begin{aligned}
& I_{i ; k}=c_{i ; k}^{*}\left[\left(w_{, x}\right)_{i ; k}^{*}\right]^{2}+c_{i ; k}^{*}\left[\left(w_{, y}\right)_{i ; k}^{*}\right]^{2}+ \\
& +2 c_{i ; k}^{*}\left(w_{, x}\right)_{i ; k}^{*}\left(v_{, x}\right)_{i ; k}^{*}+2 c_{i ; k}^{*}\left(w_{, y}\right)_{i ; k}^{*}\left(v_{, y}\right)_{i ; k}^{*}
\end{aligned}
$$

and

$$
c_{i ; k}^{*}=\left(c_{i ; k}+c_{i+1 ; k}+c_{i ; k+1}+c_{i+1 ; k+1}\right) / 4
$$$$
\left(w_{, x}\right)_{i ; k}^{*}=\left(w_{i+1 ; k+1}+w_{i+1 ; k}-w_{i ; k}-w_{i ; k+1}\right) / 2 h \quad,
$$$$
\left(w_{, y}\right)_{i ; k}^{*}=\left(w_{i+1 ; k+1}+w_{i ; k+1}-w_{i ; k}-w_{i+1 ; k}\right) / 2 h \quad,
$$

$\left(v_{, x}\right)_{i ; k}^{*}=\left(v_{i+1 ; k+1}+v_{i+1 ; k}-v_{i ; k}-v_{i ; k+1}\right) / 2 h \quad$,

$\left(v_{, y}\right)_{i ; k}^{*}=\left(v_{i+1 ; k+1}+v_{i ; k+1}-v_{i ; k}-v_{i+1 ; k}\right) / 2 h$

with the following discrete form of boundary conditions (23) and (24)

$w_{0 ; k}=w_{m+1 ; k}=0, k=0,1, \ldots, n+1$,

$$
\begin{aligned}
& w_{i ; 0}=w_{i ; n+1}=0, i=0,1, \ldots, m+1, \\
& v_{0 ; k}=u_{0 ; k}, \quad v_{m+1 ; k}=u_{m+1 ; k}, \quad k=0,1, \ldots, n+1, \\
& v_{i ; 0}=u_{i ; 0}, \quad v_{i ; n+1}=u_{i ; n+1}, \quad i=0,1, \ldots, m+1 .
\end{aligned}
$$

Instead of finding the minimum of the functional $J$ now the problem arises to find values $w_{i ; k}, i=0,1, \ldots, m+1$, $k=0,1, \ldots, n+1$ which fulfil the boundary conditions (27) and such that (25) is minimal. The minimum of (25) is found using the method of local variations [1]. The algorithm of this method is given by a sequence of iterations. It is necessary to start the algorithm with determination of some initial approximation of the values $w_{i ; k}, \quad i=0,1, \ldots, m+1, \quad k=0,1, \ldots, n+1 \quad$ such that the boundary conditions (27) are fulfilled. The iterative solution is obtained in such a way, that the iteration from the previous step is considered as the initial approximation and then it is necessary to go through all interior grid points in arbitrary order. At each grid point $(i h ; k h)$ $i=1,2, \ldots, m, k=1,2, \ldots, n$ is computed the value

$\Phi_{i ; k}\left(w_{i ; k}\right)=h^{2} \sum_{j=0}^{m} \sum_{l=0}^{n} \chi_{j ; l} I_{j ; l}$,

where

$\chi_{j ; l}=\left\{\begin{array}{ll}1, & I_{j ; l}=I_{j ; l}\left(w_{i ; k}\right) \\ 0, & I_{j ; l} \neq I_{j ; l}\left(w_{i ; k}\right)\end{array}\right.$,

which is the collection of such $I_{j ; l}$ from (25) which depends on the value $w_{i ; k}$. According (26) it is possible to rewrite (28) in the following form

$\Phi_{i ; k}\left(w_{i ; k}\right)=h^{2} \sum_{j=i-1}^{i} \sum_{l=k-1}^{k} I_{j ; l}$.

Then is changed a little the value $w_{i ; k}$ to the values $w_{i ; k} \pm p$, where $p>0$ is the given step and two corresponding values $\Phi_{i ; k}^{+}\left(w_{i ; k}+p\right), \quad \Phi_{i ; k}^{-}\left(w_{i ; k}-p\right)$ are computed. The new value of the solution at grid point $(i h ; k h)$ is chosen from the values $w_{i ; k}, w_{i ; k}+p$, $w_{i ; k}-p$ and it is this one which corresponds to the minimum value of $\Phi_{i ; k}\left(w_{i ; k}\right), \Phi_{i ; k}^{+}\left(w_{i ; k}+p\right)$, $\Phi_{i ; k}^{-}\left(w_{i ; k}-p\right)$. The iteration is finished after we have gone through all interior grid points. Then the value of $I$ is calculated for such solution and the process is repeated until the value of $I$ is decreasing. Then it is possible to continue by dividing the step.

We can prove similarly to [4] the following important theorem: 
THEOREM. Let the electrical conductivity is piecewise smooth function. Then the method of local variations is convergent for the problem (20), (19).

The program realization of the method of local variations for the problem is elaborated in the programming language Fortran 77.

\subsection{Numerical solution of the problem}

Numerical experiments are also now done from a mathematical point of view.

The numerical solutions were computed for different values of electrical conductivities of the media and for different values of the voltage potential on the boundary of the media. The following domain $\Omega=\langle 0,2\rangle \times\langle 0,1\rangle$ is considered.

For the following voltage potential

$u=\frac{1}{e^{x+y}}$

electrical conductivity

$c=e^{x+y}$

the obtained results can be seen in the Table 6. From the results it is possible to see that very small errors are obtained for a course mesh and when the number of grid points increases, errors also increase slightly but are still small.

Table 6 Numerical results for the problem (29), (30)

$\begin{array}{ccc}\text { Mesh } & \text { Error (\%) } & \begin{array}{c}\text { Number of } \\ \text { iterations }\end{array} \\ 8 \times 4 & 6.510^{-7} & 89 \\ 16 \times 8 & 1.310^{-6} & 216 \\ 32 \times 16 & 5.210^{-6} & 700\end{array}$

For another voltage potential

$u=\ln (x+1) \ln (y+1)$

the electrical conductivity

$c=(x+1)(y+1)$

as it is obvious from the Table 7 the accuracy of computation is not so good. This fact is caused by the discretization error, which is in this case greater than in previous case. From Table 7 it is seen that when the number of grid points increases, errors decrease.

Table 7 Numerical results for the problem (31), (32)

\begin{tabular}{|c|c|c|}
\hline Mesh & Error (\%) & $\begin{array}{c}\text { Number of } \\
\text { iterations }\end{array}$ \\
\hline $8 \times 4$ & $2.510^{-1}$ & 86 \\
\hline $16 \times 8$ & $8.3 \quad 10^{-2}$ & 211 \\
\hline $32 \times 16$ & $2.410^{-2}$ & 757 \\
\hline
\end{tabular}

One way how to obtain better results in this case is make computations for more grid points. Obtained results can be seen in the Table 8 . From the Table 8 can be seen that the errors decrease, however the number of iterations rapidly increase. Second way how to obtain better results is to use better discretization scheme.

Table 8 Numerical results for the problem (31), (32)

$\begin{array}{ccc}\text { Mesh } & \text { Error (\%) } & \begin{array}{c}\text { Number of } \\ \text { iterations }\end{array} \\ 64 \times 32 & 6.510^{-3} & 2466 \\ 128 \times 64 & 1.810^{-3} & 7985 \\ 256 \times 128 & 1.210^{-3} & 26857\end{array}$

For another voltage potential

$u=\frac{1}{(x+1)(y+1)}$

the electrical conductivity

$c=(x+1)^{2}(y+1)^{2}$

as it is obvious from the Table 9 the accuracy of computation is also now not so good. Also now this fact is caused by the discretization error, which is in this case greater than in the first case. From Table 9 it is seen that when the number of grid points increases, errors decrease.

Table 9 Numerical results for the problem (33), (34)

$\begin{array}{ccc}\text { Mesh } & \text { Error (\%) } & \begin{array}{c}\text { Number of } \\ \text { iterations }\end{array} \\ 8 \times 4 & 2.8 \quad 10^{-1} & 83 \\ 16 \times 8 & 6.8 \quad 10^{-2} & 201 \\ 32 \times 16 & 1.7 \quad 10^{-2} & 766\end{array}$

Also now one way how to obtain better results in this case is make computations for more grid points. Obtained results can be seen in the Table 10. From the Table 10 also now can be seen that the errors decrease, however the number of iterations rapidly increase. Second way how to obtain better results is to use better discretization scheme.

Table 10 Numerical results for the problem (33), (34)

$\begin{array}{ccc}\text { Mesh } & \text { Error (\%) } & \begin{array}{c}\text { Number of } \\ \text { iterations }\end{array} \\ 64 \times 32 & 4.310^{-3} & 2343 \\ 128 \times 64 & 1.110^{-3} & 7820 \\ 256 \times 128 & 4.910^{-4} & 25819\end{array}$

We also deal with the case when the electrical conductivity is discontinuous 
$c=2, x \leq \frac{2}{3}$,

$c=2.5, \quad x>\frac{2}{3}$.

Then for the voltage potential

$$
\begin{aligned}
& u=(x+1)+(y+1), x \leq \frac{2}{3}, \\
& u=\frac{4(x+1)}{5}+(y+1)+\frac{1}{3}, x>\frac{2}{3}
\end{aligned}
$$

as it is obvious from the Table 11 the accuracy of computation is also now not so good. Also now this fact is caused by the discretization error, which is in this case greater than in the first case. From Table 11 it is seen that when the number of grid points increases, errors decrease.

Table 11 Numerical results for the problem (35), (36)

$\begin{array}{ccc}\text { Mesh } & \text { Error (\%) } & \begin{array}{c}\text { Number of } \\ \text { iterations }\end{array} \\ 8 \times 4 & 2.1 \quad 10^{-1} & 72 \\ 16 \times 8 & 5.410^{-2} & 242 \\ 32 \times 16 & 5.510^{-2} & 859\end{array}$

One way how to obtain better results also in this case is make computations for more grid points. Obtained results can be seen in the Table 12. From the Table 12 can be seen that the errors decrease, however the number of iterations rapidly increase. Also now second way how to obtain better results is to use better discretization scheme.

\begin{tabular}{|c|c|c|}
\hline Mesh & Error $(\%)$ & $\begin{array}{l}\text { Number of } \\
\text { iterations }\end{array}$ \\
\hline $64 \times 32$ & $1.410^{-2}$ & 3054 \\
\hline $128 \times 64$ & $1.410^{-3}$ & 10629 \\
\hline $256 \times 128$ & $3.510^{-4}$ & 35049 \\
\hline
\end{tabular}

Table 12 Numerical results for the problem (35), (36)

For more numerical experiments see [5].

\section{CONCLUSIONS}

This paper is dealt with the numerical determination of voltage potential inside one and two dimensional nonhomogeneous media using values of the voltage potential on the boundary of the media.

From computed numerical examples it is seen that obtained accuracy of the computation depends on the discretization errors. For obtaining better results it is necessary to make computations for more grid points or to use better discretization scheme.

This approach is possible to generalize to the problem for numerical determination of voltage potential inside three dimensional nonhomogeneous media using variational methods.

\section{ACKNOWLEDGMENTS}

This work was supported by the Slovak Research and Development Agency under the contract No. APVV-150326.

\section{REFERENCES}

[1] ČERNOUSKO, F. L. - BANIČUK, N. V.: Variacionnye zadači mechaniki $i$ upravlenija, Moskva: Izdatel'stvo Nauka, 1973.

[2] REKTORYS, K.: Variační metody v inženýrských problémech a v problémech matematické fyziky, Praha: SNTL, 1974.

[3] BRILLA, I.: Numerical Determination of Voltage Potential Inside Nonhomogeneous Media, Proceedings of the 14th International Scientific Conference on Electric Power Engineering 2013, Kouty nad Desnou, pp. 1-6, May 2013.

[4] BRILLA, I.: Numerical Determination of Voltage Potential Inside Orthotropic Media Using Variational Methods, Proceedings of the 11th International Scientific Conference Energy-Ecology-Economy 2012, Tatranské Matliare, pp. 1-6, May 2012.

[5] BRILLA, I. - JANÍČEK, F.: Numerical determination of voltage potential inside two dimensional nonhomogeneous media using variational methods, Proceedings of the 14th International Scientific Conference Energy-EcologyEconomy 2018, Tatranské Matliare, pp. 1-6, June 2018.

Received September 13, 2018, accepted January 14, 2019

\section{BIOGRAPHIES}

Igor Brilla was born in Bratislava on 9.11.1956. He graduated from the Faculty of Mathematics and Physics, Comenius University in Bratislava in 1981 and he received RNDr. degree in 1981. He defended his CSc. degree in applied mathematics in 1988. From 1981 to 1991 he was at the Institute of Applied Mathematics and Computing Technique of Comenius University as scientific worker and now is working at the Department of Mathematics, Faculty of Electrical Engineering and Information Technology, Slovak University of Technology in Bratislava. His research activity is oriented to numerical solution in engineering and applied sciences.

František Janíček was born on 4.12.1954 in Čadca. He graduated from the Faculty of Electrical Engineering (EF) of the Slovak University of Technology (SVŠT, later STU) in 1979; in the same year he took the position of Assistant Lecturer at the Department of Power Engineering of EF SVŠT. In 1984, he obtained a Ph.D. in the field of Power Engineering at EF SVŠT. On 30 September 1999, he received the academic degree of Professor. He has been the Dean of the Faculty of Electrical Engineering and Information Technology of STU in Bratislava. He has been the Vice-Rector for 
Development of the Slovak University of Technology. Electrical Engineering and Information Technology of Now he is the head of Institute of Power STU. He is a leading expert in the area of power and Applied Electrical Engineering, Faculty of engineering. 